\title{
Vector-control response in a post-flood disaster setting, Honiara, Solomon Islands, 2014
}

\author{
Matthew Shortus, ${ }^{a}$ Jennie Musto, ${ }^{a}$ Hugo Bugoro, ${ }^{b}$ Charles Butafa, ${ }^{b}$ Alison Sioc and Cynthia Joshua ${ }^{c}$ \\ Correspondence to Matthew Shortus (email: shortusm@wpro.who.int).
}

Problem: The close quartering and exposed living conditions in evacuation centres and the potential increase in vector density after flooding in Solomon Islands resulted in an increased risk of exposure for the occupants to vectorborne diseases.

Context: In April 2014, Solomon Islands experienced a flash flooding event that affected many areas and displaced a large number of people. In the capital, Honiara, nearly 10000 people were housed in emergency evacuation centres at the peak of the post-flood emergency. At the time of the floods, the number of dengue cases was increasing, following a record outbreak in 2013.

Action: The National Vector Borne Disease Control Programme with the assistance of the World Health Organization implemented an emergency vector-control response plan to provide protection to the at-risk populations in the evacuation centres. The National Surveillance Unit also activated an early warning disease surveillance system to monitor communicable diseases, including dengue and malaria.

Outcome: Timely and strategic application of the emergency interventions probably prevented an increase in dengue and malaria cases in the affected areas.

Discussion: Rapid and appropriate precautionary vector-control measures applied in a post-natural disaster setting can prevent and mitigate vectorborne disease incidences. Collecting vector surveillance data allows better analysis of vectorcontrol operations' effectiveness.

\section{PROBLEM}

There was concern that the large populations of displaced people in evacuation centres in Honiara, Solomon Islands were vulnerable to several communicable diseases (including vectorborne diseases such as malaria and dengue) after severe flooding in April 2014. The risk for dengue transmission in the evacuation centres was considered high due to several contributory factors: the increasing dengue circulation among the general population before the floods; large populations living in close, confined conditions; locations of evacuation centres that were covered but were unscreened openair structures; and optimal environmental conditions with widespread availability of Aedes (and potentially Anopheles) breeding sites.
This paper describes the vectorborne disease risk assessment conducted in the affected areas of Honiara and Guadalcanal Province and the application of rapid response interventions to reduce the level of exposure of those living in the evacuation centres.

\section{CONTEXT}

Solomon Islands has a history of dengue outbreaks with several recorded dengue epidemics since the 1970s. ${ }^{1}$ Two dengue vectors are now present in Honiara, namely, Aedes aegypti and Aedes albopictus. The country experienced its largest documented dengue outbreak in 2013 (type 3), with nearly 8000 cases and eight deaths. Aedes aegypti re-emerged in Honiara during 2013 after last being identified in the 1980s. The even

\footnotetext{
WHO Office of the Representative in Solomon Islands, Honiara, Solomon Islands.

National Vector Borne Disease Control Program, Ministry of Health and Medical Services, Point Cruz, Honiara, Solomon Islands.

National Surveillance Unit, Ministry of Health and Medical Services, Chinatown, Honiara, Solomon Islands.

Submitted: 30 July 2015; Published: 21 January 2015

doi: 10.5365/wpsar.2015.6.3.004
} 
Figure 1. Reported dengue cases, EWARN dengue cases and rainfall during pre- and post-flood periods, March-June 2014, Honiara, Solomon Islands

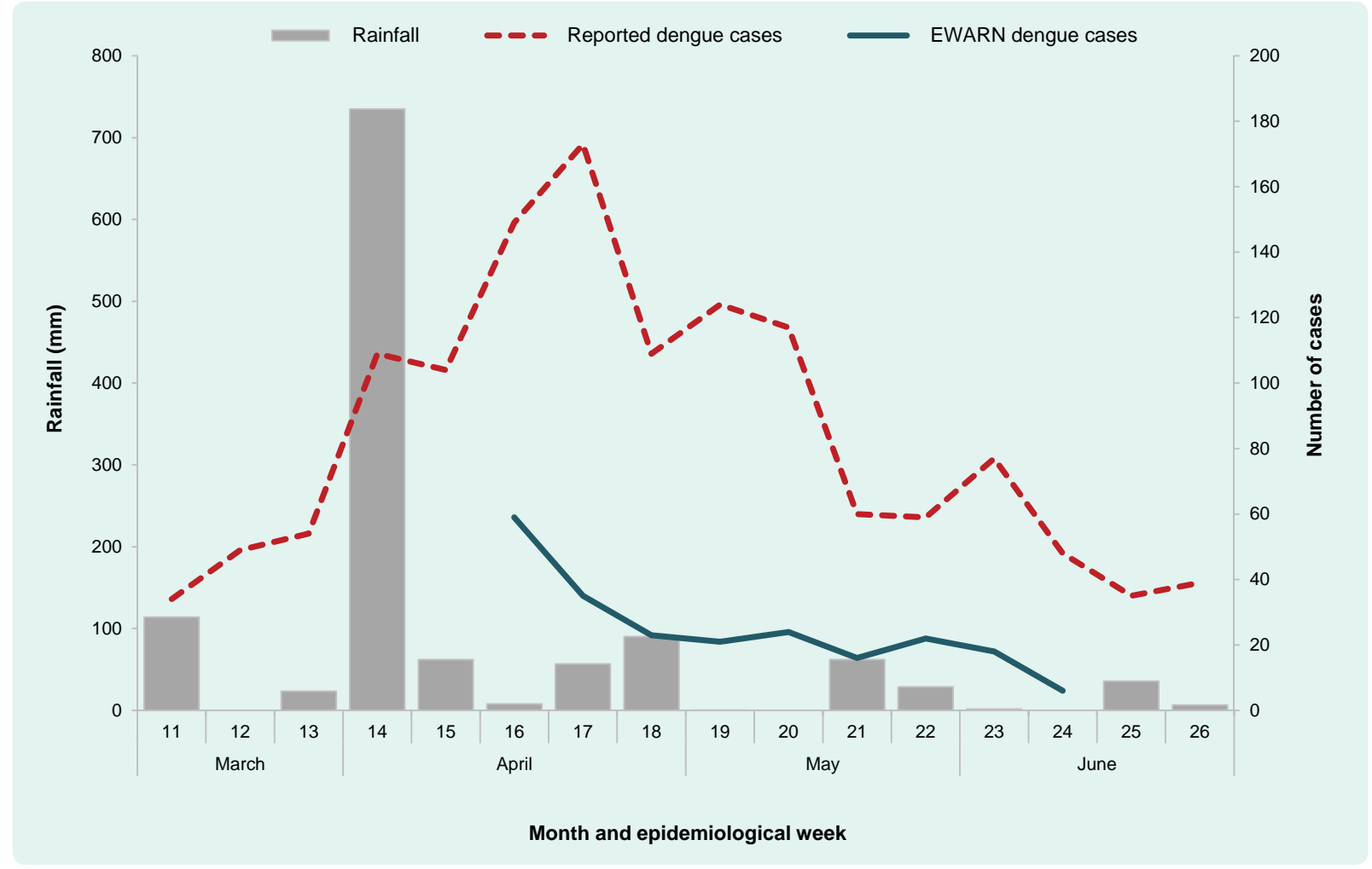

EWARN, Early Warning Alert and Response Network.

distribution of infections across age groups suggested that type 3 dengue had not circulated in the country for several years. ${ }^{2}$ Due to this absence of immunity in the population, the outbreak continued into early 2014, with cases increasing in March 2014 as environmental conditions became more favourable for vector production.

In early April 2014, a tropical depression formed a trough over Solomon Islands causing $732.5 \mathrm{~mm}$ of rain to fall between 2 and 5 April (Figure 1). This triggered severe flooding in the capital and many other provinces, especially in Guadalcanal Province. The flooding resulted in the death of 23 people and the displacement of approximately 50000 people. The majority of the affected people came from Guadalcanal Province and Honiara. At the peak of the crisis, nearly 10000 people were being housed in 31 evacuation centres within Honiara.

The Guadalcanal plains to the east of Honiara, and the peri-urban areas on the eastern and western margins of Honiara historically experience high levels of malaria transmission between March and June. Epidemic risk of both malaria and dengue in evacuation centres located in these areas was high during the April 2014 post-flood period.

\section{ACTION}

\section{Risk assessment}

As requested by the Ministry of Health and Medical Services of the Solomon Islands, a post-disaster outbreak risk assessment was conducted by the World Health Organization (WHO) four days after the flood event. A WHO epidemiologist visited the major evacuation centres, the National Referral Hospital and other health facilities in Honiara and Guadalcanal Province to assess the post-disaster epidemic risk factors, including sanitation, water quality, living conditions of displaced populations, exposure to flood water and exposure to disease vectors using standard WHO guidelines. ${ }^{3}$

\section{Establishment of Early Warning Alert and Response Network (EWARN)}

The risk assessment recommended implementing an early warning disease surveillance system to monitor 
epidemic diseases; therefore, a paper-based EWARN system was implemented after the floods. The EWARN system was an enhancement of the existing routine syndromic surveillance system, coordinated by the National Surveillance Unit (NSU), and used the same data collection methods which involves weekly visits to sentinel sites (permanent health clinics) to collect and aggregate tallies that are manually recorded by facility staff for the targeted syndromes. The existing NSU system collects weekly disease data on five syndromes (dengue-like illness, acute fever and rash, diarrhoea, influenza-like illness and prolonged fever) from four sentinel sites in Honiara and five sites in other provinces. The EWARN system collected data from an additional six sentinel sites within Honiara and 12 health facilities in Guadalcanal Province. EWARN monitored eight diseases and syndromes that included the five routine syndromes plus malaria, bloody diarrhoea and acute jaundice. Positive case detection for EWARN was based on clinical definitions of the targeted syndromes. In addition, some samples were also collected for laboratory and/or rapid diagnostic tests confirmation (for example, dengue, malaria and rotavirus).

Data for EWARN were collected weekly and analysed using Excel (Microsoft Excel, Redmond, USA) by the NSU. Thresholds were set for each of the syndromes which, if exceeded, triggered verification and investigation. The thresholds for dengue and malaria were set at "twice the average number of cases seen in the previous three weeks".

\section{Vector-control responses for high-risk transmission sites}

Precautionary preventative strategies were implemented by the National Vector Borne Disease Control Programme (NVBDCP). Interventions were primarily focused on larger evacuation centres due to limited resources. These included:

(1) minimizing exposure to adult vector activity through reducing the density and the age of adult populations by targeted application of interior residual spraying (IRS) and peri-focal spraying (active ingredient: lambda-cyhalothrin), application of ultra-low volume fog (ULV) (active ingredient: deltamethrin) and distribution of longlasting insecticide-treated nets (LLINs) (active ingredient: deltamethrin);
(2) reducing juvenile vector populations through manual removal or mitigation of all potential Aedes breeding sites in evacuation centres and applying larvicide (pyriproxyfen granules) to all other potential Aedes breeding sites and all positive Anopheles breeding sites in the eastern and western fringes of Honiara; and

(3) providing barriers between hosts and vectors through distribution of LLINs to all residents in the major evacuation centres.

\section{OUTCOME}

\section{Risk assessment}

The risk assessment revealed that several conditions were increasing the risk of communicable disease outbreaks in the community, in particular poor sanitation, limited and poor water quality, displaced populations living in dirty and densely populated evacuation centres, exposure to flood water and increased exposure to disease vectors.

Heavy rains would have flushed Aedes larvae from breeding sites while also flooding many receptacles and hatching dormant reserves of Aedes eggs. ${ }^{4}$ This could lead to increases in adult dengue vector populations within 7-10 days, and it was therefore realistic to expect dengue transmission to potentially increase within 3-4 weeks. ${ }^{5,6}$

The flood waters could also have created large tracts of suitable breeding sites for Anopheles farauti, the major endemic malaria vector species. ${ }^{7-9}$ In coastal areas where rivers and creeks had burst their banks and formed temporary pools, flood water could potentially form suitable breeding sites. Increases in mosquito productivity from these sites could be expected to take between 2-4 weeks and 6-8 weeks before affecting malaria incidence. ${ }^{8-10}$

\section{EWARN}

EWARN helped the NVBDCP to track potential epidemic outbreaks of key vectorborne diseases in Honiara and Guadalcanal Province in the wake of the floods. The EWARN system was initiated on 14 April and operated for nine weeks after the floods. Weekly reporting of geographical coverage rates varied from 
Table 1. Coverage of vector-control interventions for the estimated population who were displaced in Honiara, Solomon Islands, $2014(n=8080)$

\begin{tabular}{lccc}
\hline & LLINs & Residual spraying & ULV \\
\hline Displaced population covered (\%) & $6499(80 \%)$ & $6188(77 \%)$ & $6188(77 \%)$ \\
Material/area coverage & 4180 nets & $12062 \mathrm{~m}^{2}$ & $8780000 \mathrm{~m}^{2}$ (878 hectares) \\
Activity date range (2014) & 7 to 10 April & 9 to 15 April & 8 April to 11 June \\
\hline
\end{tabular}

LLINs, long-lasting insecticide-treated nets; and ULV, ultra-low volume.

$73 \%$ to $91 \%$. Lack of coverage was due to either flooddamaged health facilities or failure to submit reports. The EWARN system's alert threshold was triggered once for vectorborne diseases in Honiara with malaria exceeding the defined threshold in late April.

\section{Vector-control responses}

A total of 4180 LLINs were distributed to the evacuation centres, providing coverage to $6499 / 8080(80 \%)$ of the estimated displaced population (Table 1). The LLINs were considered the highest priority intervention for protection against malaria vectors, so delivery of all LLINs was completed within five days of the mass evacuations to the evacuation centres.

Residual and ULV space spraying were the next priority interventions with IRS and peri-focal spraying applied to potential indoor and outdoor mosquitoresting sites in all structures and to potential Aedes breeding sites (excluding potable water sources) at 11 of the largest evacuation centres. This provided additional protection to $6188 / 8080$ (77\%) of the estimated displaced population (Table 1). Interior wall surfaces in the evacuation centres were mainly exposed or painted dressed timber, so the encapsulated suspension formulation of the IRS chemical would have provided an effective treatment. ULV space spraying was conducted using backpack foggers at the same 11 evacuation centres. Spraying was done in the late afternoon or early evening to target the peak activity times of the two major dengue vectors. ULV space spraying was also conducted using a truck-mounted fogger (LECO 1800E, Clarke, St Charles, Illinois, USA) in high transmission suburbs/areas around Honiara, focusing on those areas having evacuation centres. During the first two weeks after the floods, all evacuation centres were treated with backpack ULVs twice a week. ULV treatments in evacuation centres were stopped as residents were relocated. Ongoing truck-mounted ULV treatments targeting high transmission areas, which were identified using data from the dengue line list, were applied weekly. A total of 878 hectares were treated with ULV from 8 April to 11 June (Table 1 ).

The responses successfully minimized the exposure of at-risk displaced populations to vector activity, reduced juvenile vector populations and provided barriers between hosts and vectors.

\section{DISCUSSION}

The number of dengue cases detected by EWARN was generally lower than the reported dengue cases. This may be because the EWARN site at the National Referral Hospital included only cases from the emergency department but not admitted cases. However the general trend of dengue transmission was reflected in both systems (Figure 1). While the number of malaria cases exceeded the threshold set within EWARN in epidemic week 18 (last week of April 2014), this incidence level was consistent with the annual pattern of malaria transmission in Honiara over the past three years as reported in the national routine malaria information system (Table 2). This result indicates that although EWARN is sensitive for monitoring disease incidence against a baseline projection, it can be misleading if the baselines are set without referencing the historical disease trends.

The displacement of large populations of people into evacuation centres plus flood waters potentially generating an increase of disease vectors was a combination of circumstances that presented a clear vectorborne disease epidemic risk. While it is not able to be determined from the disease surveillance data the level of protection that was afforded to these at- 
Table 2. Historical trend of March to June clinical malaria cases in Honiara, 2012-2014 and EWARN malaria cases in Honiara, Solomon Islands during the same months in 2014

\begin{tabular}{|c|c|c|c|c|}
\hline & \multicolumn{3}{|c|}{ Clinical malaria cases* } & \multirow{2}{*}{$\begin{array}{l}\text { EWARN malaria cases }{ }^{\dagger} \\
2014\end{array}$} \\
\hline & 2012 & 2013 & 2014 & \\
\hline March & 770 & 930 & 637 & - \\
\hline April & 617 & 836 & 705 & 326 \\
\hline May & 768 & 772 & 605 & 398 \\
\hline June & 501 & 461 & 763 & $174^{\ddagger}$ \\
\hline \multicolumn{5}{|c|}{ EWARN, Early Warning Alert and Response Network. } \\
\hline \multicolumn{5}{|c|}{$\begin{array}{l}\text { * Monthly clinical malaria cases data were extracted from the routine malaria information system, National Vectorborne Disease } \\
\text { Control Programme. }\end{array}$} \\
\hline \multicolumn{5}{|c|}{${ }^{\dagger}$ Aggregated weekly EWARN data for clinical malaria cases. } \\
\hline
\end{tabular}

risk populations, it is likely that the rapid precautionary vector-control measures that were put in place prevented larger numbers of vectorborne-disease cases in the evacuation centres. Although a high level of intervention coverage was achieved, several problems were faced by the teams when implementing these interventions. Data availability on evacuation centres' populations and locations and the coordination of emergency services were inherent problems, especially immediately after the floods. These issues affected the operational planning and quantification of interventions required for vector control and other essential emergency services. Delivering emergency control interventions to displaced populations was more efficient when the lists of designated evacuation centre locations and their populations were provided. The information is extremely important for rapid and effective emergency public health interventions.

Unfortunately, no vector surveillance data was collected during this period, which would have complemented the disease surveillance and intervention data and allowed better guidance and analysis of the vector-control operations. ${ }^{11-13}$ This highlights the importance of collecting routine data on vector populations as well as conducting pre- and post-control surveillance of targeted vectors. Since the floods, a routine adult Aedes surveillance programme is now being conducted in Honiara with BG-Sentinel traps (BioQuip Products, Inc., Rancho Dominguez, CA, USA) set at 20 sentinel sites in the city. Data are collected and analysed every two weeks. Monthly monitoring of larval density in major Anopheles breeding sites close to high population areas around Honiara is also conducted. These activities can help inform the NVBDCP on the effectiveness of their vector-control interventions.

The public health responses to the 2014 Honiara floods highlighted several important lessons in providing vector-control interventions in a disaster setting. Rapid epidemic disease risk assessment and ongoing disease alert networks can provide evidence to prioritize public health interventions. Also it is necessary to implement the interventions in a timely and effective manner. Furthermore, it is important for public health officials to work closely with the disaster management bodies and to share vital information on vulnerable populations so as to help better targeted interventions.

\section{Conflicts of interest}

None declared.

\section{Funding}

None.

\section{Acknowledgements}

The authors would like to acknowledge the assistance by the Solomon Islands Department of Health and Medical Services staff as well as the Office of the WHO Representative in Solomon Islands staff during the manuscript writing stage. 


\section{References}

1. Darcy A et al. Solomon Islands dengue seroprevalence studyprevious circulation of dengue confirmed. Papua New Guinea Medical Journal, 2001, 44:43-47. pmid:12418677

2. Nogareda $F$ et al. Ongoing outbreak of dengue serotype-3 in Solomon Islands, January to May 2013. Western Pacific Surveillance and Response Journal, 2013, 4:28-32. doi:10.5365/wpsar.2013.4.2.013 pmid:24319611

3. Rapid risk assessment of acute public health events. Geneva, World Health Organization, 2012 (http://apps.who.int/iris/ bitstream/10665/70810/1/WHO_HSE_GAR_ARO_2012.1_eng. pdf, accessed 4 December 2015).

4. Dieng $\mathrm{H}$ et al. The effects of simulated rainfall on immature population dynamics of Aedes albopictus and female oviposition. International Journal of Biometeorology, 2012, 56:113-120. doi:10.1007/s00484-011-0402-0 pmid:21267602

5. Dibo MR et al. Study of the relationship between Aedes (Stegomyia) aegypti egg and adult densities, dengue fever and climate in Mirassol, state of São Paulo, Brazil. Memorias do Instituto Oswaldo Cruz, 2008, 103:554-560. doi:10.1590/ S0074-02762008000600008 pmid:18949325

6. Hii YL et al. Forecast of dengue incidence using temperature and rainfall. PLoS Neglected Tropical Diseases, 2012, 6(11):e1908. doi:10.1371/journal.pntd.0001908 pmid: 23209852

7. Bugoro $\mathrm{H}$ et al. Influence of environmental factors on the abundance of Anopheles farauti larvae in large brackish water streams in Northern Guadalcanal, Solomon Islands. Malaria
Journal, 2011, 10:262. doi:10.1186/1475-2875-10-262 pmid:21910907

8. Charlwood JD, Graves PM, Alpers MP. The ecology of the Anopheles punctulatus group of mosquitoes from Papua New Guinea: a review of recent work. Papua New Guinea Medical Journal, 1986, 29:19-26. pmid:3463014

9. Paik $\mathrm{YH}$. Influence of stagnation of water pathways on mosquito population density in connection with malaria transmission in the Solomon Islands. The Japanese Journal of Experimental Medicine, 1987, 57:47-52. pmid:3626094

10. Gagnon AS, Smoyer-Tomic KE, Bush AB. The El Niño southern oscillation and malaria epidemics in South America. International Journal of Biometeorology, 2002, 46:81-89. doi:10.1007/ s00484-001-0119-6 pmid:12135203

11. Dengue guidelines for diagnosis, treatment, prevention and control, new edition. Geneva, World Health Organization Special Programme for Research and Training in Tropical Diseases, 2009 (http://www.who.int/tdr/publications/documents/denguediagnosis.pdf, accessed 4 December 2015).

12. Yang G-J, Brook BW, Bradshaw CJA. Predicting the timing and magnitude of tropical mosquito population peaks for maximizing control efficiency. PLoS Neglected Tropical Diseases, 2009, 3:e385. doi:10.1371/journal.pntd.0000385 pmid: 19238191

13. Masuh $\mathrm{H}$ et al. Aedes aegypti (Diptera: Culicidae): monitoring of populations to improve control strategies in Argentina. Parasitology Research, 2008, 103:167-170. doi:10.1007/s00436-0080945-0 pmid:18344071 